\title{
S1 Figure:
}

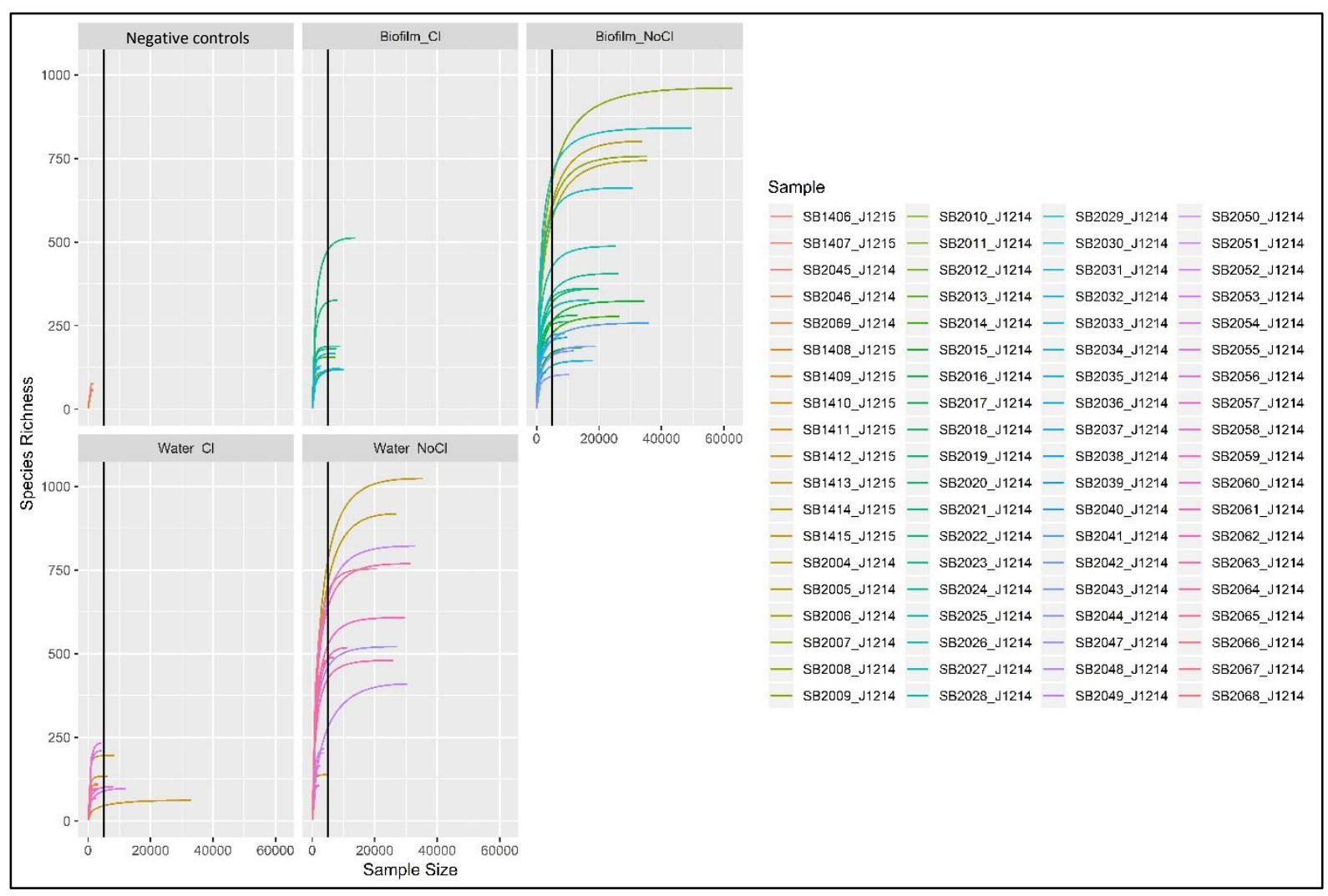

S1 Figure Legend: Rarefaction curves of 16s rRNA gene sequences of all samples grouped by negative controls (top left), chlorinated biofilm (top middle), non-chlorinated biofilm (top right), chlorinated water (bottom left) and non-chlorinated water (bottom right). The vertical line indicates the sequence number cut-off at 5,000 sequences. Samples with less than 5,000 sequences were excluded and the remaining samples rarefied to 5,259 sequences for subsequent ordinations and PERMANOVA analysis. 